\title{
Tools, Architectures and Techniques for Monitoring Energy Efficiency in Computer Networks: State of the Art Survey
}

\author{
Mwavu Rogers \\ Dept. of Information \\ Technology \\ Mbarara University of Science \\ and Technology
}

\author{
Mugonza Robert \\ Dept. of Computer Science \\ Mbarara University of Science \\ and Technology
}

\author{
John Businge, $\mathrm{PhD}$ \\ Dept. of Computer Engineering \\ Mbarara University of \\ Science and Technology
}

\begin{abstract}
Energy efficiency in data center networks is increasingly becoming a gold standard for implementers. Energy costs are becoming high while performance is highly demanded. And while efforts are directed to the design of energy efficient networks, a number of open issues require addressing including: - scalability, fault tolerance, efficiency in performance though their presence does not outweigh the benefits of networks energy-aware. Truly, we observe that improvements in computer networks hardware performance comes with increasing energy consumption. To address these complementary challenges, this paper technically surveys existing, approaches and techniques (virtualization, energyaware routing, among others), tools, and architectures employed in energy efficient computer networks. We have also provided a thorough review of data centre networks: server centric, switch centric and dual centric architectures with a view to inform developers and implementers of the overhead given architectural choice, identified challenges and opportunities. We conclude upon the survey that dual centric computer networks architectures have various nice properties for practical computer networks and provide flexible choices in designing of computer network architectures.
\end{abstract}

\section{General Terms}

Tools, architectures and techniques for monitoring energy efficiency in computer networks.

\section{Keywords}

Energy efficiency, Tools, Architectures, and Techniques and computer networks.

\section{INTRODUCTION}

The demand for energy efficient computing has of recent become high especially in computer networks. This is because each improvement in computer networks hardware performance comes at increasing energy consumption costs [1]. More so, while advancements [2] and [1] have led to faster computer processing, it is also observed that better pricing has increased ownership of computers. The efficiency in processing is attributed by the type, speed of inherent processor and the device's memory. However, the need for better performance has not been vigilant on the resulting energy costs. For instance, recent improvements in microprocessor clock rates of up to $400 \mathrm{GHz}$ (i7 intel processors) has raised the energy costs to up to above $70 \mathrm{~W}$ [3].

And while energy monitoring has become a vital issue, many factors attribute to the increasing energy in computer networks. Mainly, first and foremost, the increased energy waste in desktop computers, focusing at both environmental and Technical aspects. For example, the work of Bray and Megan [4] argues that in most organizations, desktop computers have a high energy consumption rate in personal offices and networks environments with $40 \%-60 \%$ of the energy consumption. This condition has given rise to many computing paradigms (green computing, cloud computing, etc.) that aim at minimizing the rate of heat dispersion and environmental degradation [5]. Desktops today are energy hungry due to the kind of work they do like; multitasking, multimedia and communication. Thus, these all intensify the energy consumption problem.

Secondly, the increased demand and use for computers. Today most of the organizations are automating most of their businesses for a competitive advantage. And while we observe their increased demand in businesses, the adverse effect is towards expenditure on the energy consumed. The high energy consumption is attributed to the unpredictable computing behaviors by the users [6]. This raises an insight towards energy wastage in such a similar environment and thus, a significant waste of money.

Managing computer use may significantly result into reduced energy consumption by $80 \%$ than when not managed [4]. On the other hand, an efficient device consumes energy proportional to its output or utility. Thus, an idle or lightly utilized PC should not consume the same energy as one that is highly utilized.

Other factors also exist that make energy consumption an important metric in computer networks. These may include:-.

\section{a) Fast growth of the Internet:}

The advent of the internet and its increased social interest has caused many people to increasingly relying on electronic data [7]. There has been a rapid adoption of internet communications and media, computerization of business processes and applications, legal requirements for record retentions and disaster recovery. All these have led to the rapid growth in the size and number of data centers and computer networks. Specifically, interests for video and music downloads, on-line gaming, social networking site visits and VoIP seem to be key drivers. The work done by Wong [8] argues that the industry use of the internet has increased. Internet usage is growing at more than 10 percent annually leading to an estimated $20 \%$ compound annual growth rate (CAGR) in computer networks. 
b) The increased need for cooling requirements in computer networks.

The work of [9],[10] and[11] show that an increasing equipment power density has led to the need of energy efficiency. Although advances in server central processing units (CPUs) have in some cases enabled higher performance with less power consumption per CPU, overall server power consumption has continued to increase as more servers are installed with higher performance power-hungry processors with more memory capacity. The increase in server power density has led to associated increase in data center and computer networks heat density. This is because servers require approximately 1 to 1.5 watts of cooling for each watt of power used [12],[13] and[14] The ratio of cooling power to server power requirements will continue to increase as computer networks and data center server densities increase.

For example Foley [15] also shows that Companies such as Google, Microsoft, and Yahoo with the need for large data centers may not be able to find cheaper power in major American cities however have to resort to establishing data centers in the pacific where they have direct access to lowcost hydroelectric

The work of Forrest and William [16] shows that the low server utilization rates in data center and computer networks efficiency is a major problem in terms of energy use. The server utilization rates average 5-10 per cent for large data centers. Low server utilization means companies are overpaying for energy, maintenance, operations support, while only using a small percentage of computing capacity[17]

c) The growing awareness of information technology's impact on the environment.

Studies by [16] and [18] have increasing show cased how the growing awareness of information technology's impact on the environment have increased the need for energy efficiency in computer networks and datacenters. Carbon emissions have been observed to be directly proportional to energy usage in data centers with approximately 44 million servers worldwide consuming $0.5 \%$ of all electricity. Data centers in the U.S use more than $1 \%$ of all electricity [18] with their collective annual carbon emissions of 80 metric megatons of $\mathrm{CO}_{2}$ are approaching the carbon footprint of the Netherlands and Argentina [16]. And while Carbon emissions from industrial operations are expected to grow at more than $11 \%$ per year to 340 metric megatons by 2020 , the carbon footprint from manufacturing IT products is largely unaccounted for by IT organizations [16].

Interestingly, promise for reduction in emissions can prolong human life. The International Energy Agency (IEA) estimates that 72 percent of the global decrease in $\mathrm{CO} 2$ emissions between 2010 and 2020 will come from energy efficiency improvements; efficiency gains continue to drive GHG reductions with 44 percent of the reduction in GHG emissions from 2010 to 2035 coming from efficiency gains.

\section{ENERGY EFFICIENCY}

Energy efficiency is key towards achieving a low-carbon economy. Lowing energy use and cutting down on its waste, can reduce energy costs, make our energy system more sustainable, and drive down greenhouse gas emissions [16]. Having an energy efficient computer network system can reduce energy bills for computer networks,

\subsection{Energy-Efficient computer networks}

In large scale computer networks like data centers, energy efficiency has become a major priority[9]. ICT alone is responsible for $2 \%$ of the global carbon footprint production and this figure is expected to grow significantly in the future [19]. The increase in energy consumption in datacenter will definitely result in more emission of greenhouse gases, a main contributor to global warming.

Energy consumption has always been a critical concern for laptop and hand-held devices, as these devices generally run on batteries and are not connected to the electrical power grid [20]. With this, diminutive focus has been put on desktops because they are connected directly to the power grid. This has resulted into energy wastage because desktop computers consume energy in all kinds of states. This is to say, active, sleep and idle states. As a result, a shift in research has been directed towards low-energy design and conservation [20]. Studies from different researchers [4] and [21] argue that an average modern desktop computer uses $30 \%$ - $40 \%$ of its energy while idle.

Power management reduces the energy consumed by desktops while they are not in use. This represents a clear opportunity for saving money on energy costs.

Energy is a strategic resource, the efficient usage and management of this resource should always be a priority. Without energy management, mission critical applications would be starved, disrupting services that impact the operational activities. As such, this study sought to illuminate the energy management strategies that can be employed to reduce energy consumption in computer networks.

\section{COMPUTER NETWORK ARCHITECTURES AND POWER CONSUMPTION}

The architectural choice for a network is very important as it impacts on the overall efficiency of the computer network. It also directly reflects on its scalability, cost, fault-tolerance, agility and power consumption [22]. The energy consumption of different computer networks with emphasis on energy requirements with respect to their transmission capability has been studied[23]. Many conventional computer networks are designed using a tree-like topology. A typical example of this topology is the three-tier topology proposed in[22]. Here, the tree's leaves (end-nodes or servers) are connected to Top-ofRack (ToR) switches and these (ToR) switches are further connected to aggregation switches which in turn are connected to core routers at the root of the tree.

However, this topology has suffered numerous drawbacks such as scale, capacity, reliability, utilization and energy budget [24].With these, efforts have been dedicated to address some of them and thus, various architectures have appeared [25], [26] and [27]. These architectures can be grouped in three forms: - (i) Switch-centric, (ii) Server-centric and (iii) Dual centric.

\subsection{Switch Centric Architectures}

A switch-centric typically consists of a multi-level tree of switches to connect the end servers (typically two or three levels). Switch-centric based networks are widely adopted and implemented in today's tera scale computer networks. They are able to support communications between tens of thousands of servers. Take a conventional three-level switch-based network as an example, the ToR switches have a set of $1 \mathrm{Gbps}$ Ethernet ports and are responsible for transferring packets 
within the rack; layer two aggregation switches have 10Gbps links to interconnect ToR switches, and these layer-2 switches will be connected by a more powerful switch when more hierarchy structure are applied. In switch-based network architectures, the bottleneck is at the top level of the tree. Such bandwidth bottleneck is often reduced by employing more powerful hardware at the expense of high-end switches. Such architecture can take-up forms like:- fat-tree, portland, VL2 (Virtual Layer 2) and one-tier Qfabric though affected by a number of factors including: Oversubscription, agility, load balancing and high power consumption.

\subsubsection{Fat Tree}

Fat-Tree networks were first proposed in [25] with the network as a tree, and processors connected to the bottom layer. The distinctive feature of a fat-tree is that for any switch, the number of links going down from it to its siblings is equal to the number of links going $u p$ to its parent in the upper level. Therefore, the tree get "wider" towards the top and with a switch in the root of the tree having most links compared to any other switch below it. The architecture has interconnected racks of servers in a three-layer topology (edge, aggregation and core) as shown in figure-1.

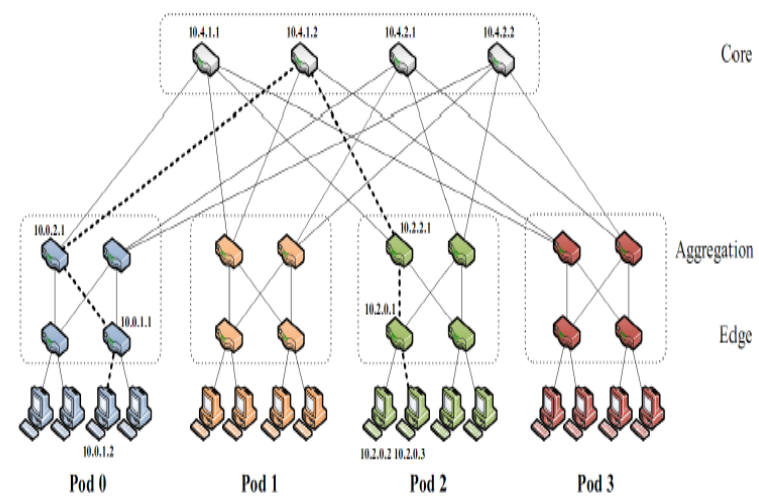

Figure- 1: The Fat-Tree Topology[25]

Each pod consists of $(\mathrm{k} / 2)$ servers \& 2 layers of $(\mathrm{k} / 2) \mathrm{k}$-port switches with each edge switch connecting to $(\mathrm{k} / 2)$ servers \& $(\mathrm{k} / 2)$ aggregate.

\subsubsection{VL2 (Virtual Layer 2)}

The work of [28] proposed VL2 as a solution to critical issues in conventional computer networks including oversubscription, agility and fault tolerance as illustrated in Figure-2 below.

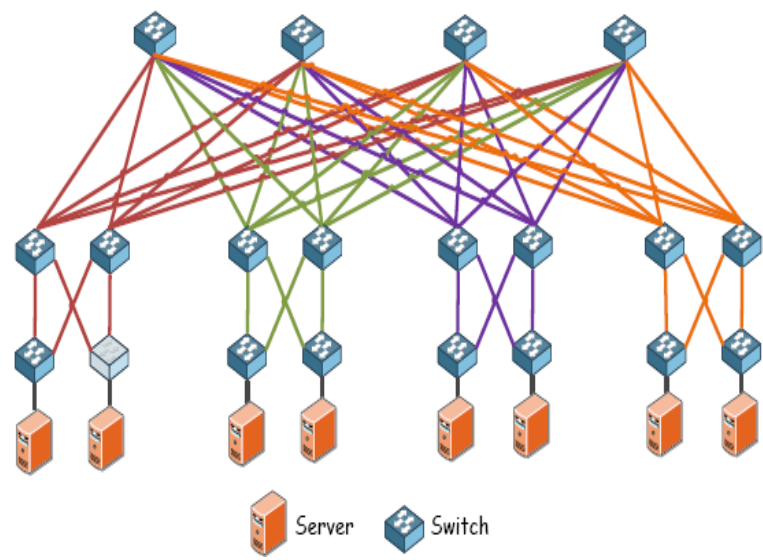

Figure 2: VL2 Computer network architecture[25]
Virtual Layer -2 (VL2) is similar to the three-tier architecture datacenter network (DCN) proposed by Cisco, except that, it implements a close topology (low cost ASICs) between core and aggregation layers to provide a multipath and rich connectivity between the two top tiers. The architectural design enhances the availability and reliability of the network, especially in the presence of link or hardware failures by employing Valiant Load Balancing (VLB) to evenly load balance traffic flows over the paths using Equal Cost Multi Path (ECMP). It employs TCP for end to end congestion control. The above characterization enables its implementation on low cost switches due to ECMP used for packet forwarding and link state routing for topology updates. More of this architecture is further discussed in the comparison section below.

\subsubsection{Portland}

Similar to Virtual Layer-2 topology, Portland, a data Centre architecture designed based on the Fat-tree networks contributing network layer 2 and 3 protocols with the ability to offer flexibility, efficiency, fault tolerance, and manageability in data centers. These are Ethernet-compatible routing, forwarding, and address resolution protocols required for data center deployments [29].

Compared to VL2, Portland is a triple -layered architecture including: Edge, Aggregation and Core. They further may differ along the ways of associating and separating names from locators, but however resemble at providing agility among services running on multiple Virtual machines (VMs). Portland imposes additional requirements on the switch software and hardware unlike VL2 where implementation only takes place in the server network stack while for load balancing within VMs, the architecture employs flow hashing in Equal Cost Multiple Path (ECMP).

\subsubsection{One-Tier Fabric Architecture}

Flattening three-tier tree structure to one tier fabric is an existing solution proposed for modern data center and computer networks architectures [25]

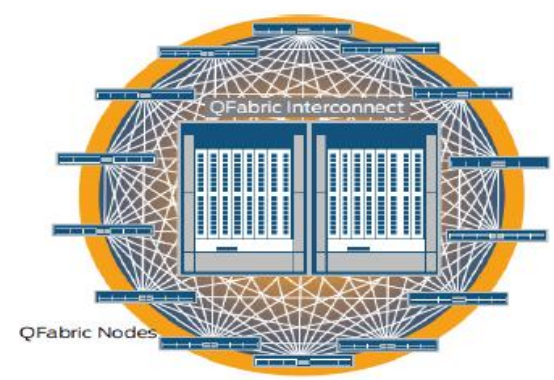

Figure 3: Qfabric architecture[25]

The Qfabric single logical switch has an added value to computer networks since it reduces the complexity, operational cost, cooling cost, occupied floor space and power consumption. The Qfabric supports high speed server to server connectivity with low latency which makes it an attractive structure for modern data centers hosting delay sensitive applications. It also smoothens the process of virtualization among servers within the data center leading to great energy savings.

\subsection{Server-Centric Architectures}

The server centric architectures use servers as relay nodes to each other. These are vital for traffic forwarding including schemes like Bcube, Dcell, Ficonn among others. Server 
centric architectures raise an implementation advantage over switch centric architectures with the ability to provide high capacity and support for all types of traffic, important for the intensive computing applications with very low delays [22].

\subsubsection{BCube}

BCube design consists of servers equipped with multiple network ports connecting multiple low cost mini switches [30]. In BCube, servers are not only hosts but they also act as relay nodes for each other and take part in traffic forwarding through multiple parallel short paths between any pair of servers. The design as observed in figure- 4 is driven by demands for intensive computing and higher bandwidth requirements to support applications with different traffic patterns such as one to one, one to many, one to all and all to all. The design provides for fault tolerance and load balancing and while requiring lower cooling and manufacturing cost.

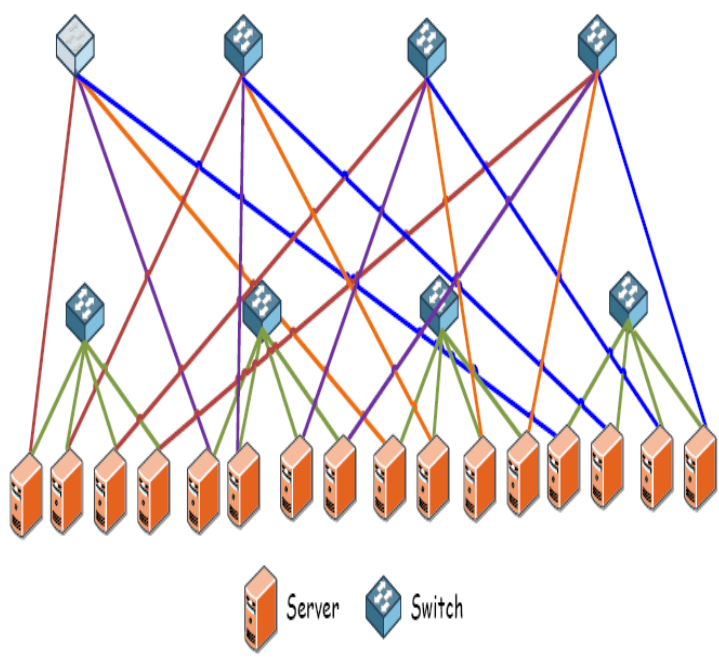

Figure 4: The BCube Topology[30]

BCube employs source routing protocol like Bootstrap Router (BSR) with Intermediate System - Intermediate System (ISIS) scale to thousands of servers. BSR can utilize high multipath capacity and load balancing to all traffic automatically. With BSR, the source server controls the selection of the path without coordination with intermediate servers which is only responsible for forwarding received packets based on information obtained from the header. BSR probes the network to select the best path which eliminates the need of frequent link state broadcasting which is not scalable since the network consists of 1000 s of servers.

\subsubsection{DCell}

Proposed as a scalable and fault-tolerant data Centre architecture, uses servers equipped with multiple network ports and mini-switches to construct its recursively defined architecture [26]. Its distributed nature of the links among servers draws the architecture advantageous as presented in figure-5.

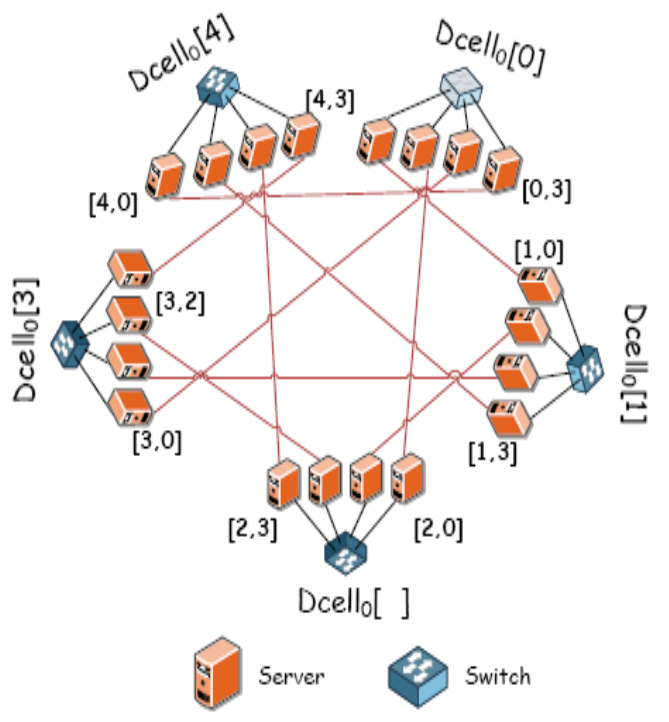

Figure 5: DCell computer network Topology[30]

DCell provides twice through-put compared to the conventional tree-based structure for Map Reduce traffic patterns. DCell is a scalable network structure which can be expanded gradually without a need for rewiring or changing addresses. And to achieve scalability and fault tolerance, the design employes a fault torelant algorithm to accommodate millions of servers while exploiting its structure with fault tolerance capability in the presence of various types of failures such as links, servers. And due to its rich physical connectivity among servers and switches, the cost for the computation and aggregation switches is lowered.

\subsubsection{Monsoon}

Monsoon, a mesh-like architecture for "cloud"-services, uses commodity switches to reduce the cost and allows powerful scaling over to 100,000 servers [27]. Performance in this architecture is ensured by employing Valiant Load Balancing (VLB). Routing between servers on the server node (source) will require two kinds of information: (a) a list of MAC addresses: to handle the destination address and (b) a list of MAC addresses of Top-of-Rack switches that servers are linked to. It thus uses the Monsoon agent to replace the userlevel Address Resolution Protocol (ARP) at every server to obtain the address information from the Monsoon directory server (maintains the server addresses to a list of Top-of-Rack switches and server MAC addresses), encapsulating each and every packet released. In a data Centre, the Monsoon design employs mechanisms for load spreading so that workload is efficiently distributed. The workload spreading is thus achieved by creating a Virtual IP shared by server pool within the architecture.

\subsection{Dual Centric Architectures (DCAs)}

Dual centric architectures exhibit features of two typical architectures: FSquare ${ }^{1}$ and FRectangle, which are entirely based on a concept of folded Clos topology [45]. While we observe features of small diameter and large bisection bandwidth, FSquare poses challenges with energy consumption. Unlike the FSquare, the FRectangle architecture significantly reduces the energy consumption per server, at a sacrifice of performances; thus, a larger diameter and a

\footnotetext{
${ }^{1}$ FSquare architecture: - a high performance computer network architecture with a small diameter and large bisection bandwidth.
} 
smaller bisection bandwidth. Observing these features of both architectures, dual centric architectures are a hybrid of both FSquare and FRectangle, hence these characteristics render them suitable for practical data centers, and provide flexible choices in designing computer architectures.

Further analysis of dual centric architectures indicates that while advancement in servers is towards use of specialized hardware for packet forwarding, and vendors providing more programmable switches via software defined networking, switches and servers will be developed with both packet forwarding capability and routing intelligence.

\subsection{Comparison of Data Center Architectures}

In this section, the researchers provide a comparison of data center architectures.

First we shall look at a fat-tree which enables the use of cheap service network components for the architecture. Fat tree has all its switching elements on the network looking alike. It also provides a path to the end hosts that uses the full bandwidth. In terms of the fault-tolerance, fat tree provides gracefully degraded performance, making it greatly outperform the tree structure. It develops a failure broadcast protocol to handle two groups of link failure between: (i) the lower- and upperlayer switches, and (ii) the upper layer and core switches. Fattree is also much more cost eff ective than the tree structure since it requires no expensive high-end switches and routers. DCell is an alternative proposal that adopts "direct" recursively defined interconnection topology. In DCell, servers in identical layers are fully connected, which makes it more scalable than fat tree. However, incremental development is a strenuous mission for DCell due to the significant cabling complexity. In addition, traffic imbalance could be a severe obstacle to considering DCell as a primary choice. BCube is the first representative Modular data center. It packs sets of servers and switches into a standard 20- or 40feet shipping-container and then connects diff erent containers through external links. Based on DCell, BCube is designed to support various traffic loads and provide high bisection bandwidth. Load balancing is an appealing advantage of BCube compared to DCell. MDCube.

The BCube uses only commercial-off-the-shelf (COTS) switches and commodity servers. Each server has small number of network ports that connect to mini-switches. The routing intelligence is left for the server. The authors claim Clos topology based solutions, such as Monsoon, VL2 and Fat-tree do not support one-to-many (one-to-one, one-toseveral and one-to-all) well, in contrast to BCube. In addition, result show that BCube offers more graceful performance degradation than typical network architectures.

In section 3.2.3 the researchers show the similarity between Monsoon and VL2 which requires a directory service and server agent for VL2 addressing and routing. Also, it seems VL2 requires changes to servers' network stacks to enable VL2 addressing and routing design. key concepts in VL2 addressing and routing are application-specific addresses (AAs) and location-specific addresses (LAs) that are used to separate server name from locations, thus providing agility. LAs are assigned for all switches and interfaces, while AAs are only used in applications. VL2 design principle is to allow implementation on existing hardware, so that VL2 could be taken in use even today.

The tree structure presents the advantages of ease-of-wire but is limited by poor scalability. It is well known that tree-based architectures are vulnerable to link failures between switches and routers and therefore fault-tolerance is poor. Fat-tree solves this problem to some extent by increasing the number of aggregation switches but the wiring become much more complex. Multipath routing is eff ective in maximizing the network capacity such as the Two Level Table, hot-spotrouting used by VL2, and location discovery-protocol (LDP) by Portland. To cope with the tremendous workload volatility in computer networks, fat-tree adopts VLB to guarantee the balance among diff erent traffic patterns. In terms of the faulttolerance.

\section{TECHNIQUES USED FOR ENERGY EFFICIENCY}

Energy efficiency or efficient use of energy relates all approaches for managing increasing costs in energy consumption. In data centres unlike other systems, energy costs have been observed to grow due to a number of factors including cooling, domant running of the centre IT without any workload. The technical survey made on data centres discusses a number of techniques that have been observed in use in data centres like virtualization, energy grid design for the data centre and the device's energy consumption cycles, energy-aware routing, rate adaptation, and voltage scaling among others.

\subsection{Virtualization}

Virtualization is considered a key enabler for machine consolidation through its recognizable role in the quest for green IT, a case for data centres [31]. The concept enables creation of Virtual Machines (VMs), encapsulating the physical machines enabling scalability and access to massive storage and computational energy for resource intensive applications. As a technique, it enables services to be moved between servers and have multiple VMs which can serve different applications multiplexed to share one server. Knowing that idle servers consume about $66 \%$ of energy at their peak time and underutilization of data centre resources averaged at $30 \%$ of the entire resources [24], servers' statistical multiplexing can be achieved through providing a seamless connectivity to merely one switch. The shutting down underutilized servers and migration of $\mathrm{VMs}$ to consolidate workloads on a set of servers could definitely lead to a great energy saving in data centers. However, many barriers[32] including:- VLANs, access lists (ACLs), broadcast domains, and Load Balancers (LB) were obstacles and prevented researchers and industries from immediate implementation of VM migration(agility) on conventional data centers. The static network assignment between servers and services in conventional data centers prevent idle servers from being assigned with overloaded services thus resulting in underutilization of resources [24].

\subsection{Energy Grid Design for the Data Centre and the device's energy}

Architectures and their styles may influence the energy consumed by the individual computing devices and ultimately the entire network[23]. Different topologies covering switch centric and server centric have been studied and simulated using energy consumption values of switches available in the market (Cisco and D-link). The energy consumption of a server's port was measured at $3 \mathrm{~W}$. And compared to architectures: DCell, BCube, Fat-tree, balanced tree architectures with comparable number of servers studied in [23] consumes less energy with limited packet transmission. DCell and BCube may appear to consume relatively similar amount of energy for small sized data centers consisting of 
about 2500 servers. However, Bcube consumes more energy for larger data center if the number of servers is to be increased to more than 5000. On the other hand, Fat-Tree structure topology was found to have moderate energy consumption values between Dcell and Bcube.

\subsection{Energy-Aware Routing}

The objective of energy aware routing is to save energy consumed through putting idle devices on sleep or shutting them down and using few network devices to provide routing with no sacrifice on network performance. Network devices consume $20 \%-30 \%$ of the energy of the whole data center[33]. In [34], an energy aware routing model was proposed and described as ERP-1: $(\mathrm{G}, \mathrm{T}, \mathrm{K})$, where $\mathrm{G}$ is the topology, $\mathrm{T}$ is the traffic matrix and $\mathrm{K}$ is a predefined threshold of network throughput. The objective is to find a routing for a specific topology where the total number of switches involved in the routing can sustain a network throughput that is equal to or higher than the defined threshold.

\subsection{Dynamic Voltage/Frequency Scaling (DVFS)}

Server's energy consumption can also be reduced by the frequency and voltage scaling. The frequency and voltage scaling represents another method to reduce servers' energy consumption, where there is a relation between voltage/frequency and the energy consumed as described by S. Herbert and D. Marculescu [44] :

$\mathrm{P}=\mathrm{V} 2 *_{\mathrm{f}}$, ( $\mathrm{f}$ is the frequency, $\mathrm{V}$ is the voltage and $\mathrm{P}$ is the energy).

With this approach, the servers' memory, bus, I/O resources and disks energy consumptions are not affected since they do not rely on the CPU frequency. However, the cost on energy consumed by the server can be significantly reduced through reducing frequency or voltage supplied to the processing chips [35]. In order to implement the DVFS technique on computing devices such as servers, hardware support for Advanced Configuration and Energy Interface (ACPI) energy management is required. The ACPI has four modes of energy states: G0 for energy-on, G1 for partial sleeping that subdivides into four states, G2 is for soft-off except with having the Energy Supply Unit (PSU) still supplying energy and G3 for energy-off state [36].

\subsection{Rate Adaptation in Networks}

Similar to the servers, DVS can be applied to links and switches to reduce energy consumption. With respect to traffic patterns and link utilizations, data rate can be reduced by applying DVS on transceivers and ports. The energy consumed by a switch can be defined as proposed in[35]

$P_{\text {switc } h}=P_{\text {chasis }}+n_{\text {linecards }}+P_{\text {linecard }}+\sum_{i=0}^{R} n_{\text {ports }, r}+P_{r}$

Where $\mathrm{P}_{\mathrm{r}}$ is the energy consumed with respect to rate.

An Ethernet link consumes 2-4W when operating at $100 \mathrm{Mbps}-1 \mathrm{Gbps}$ and can consumes 10-20W when operating at $10 \mathrm{Gbps}$, lowering the operating data centre rate could have a dramatic effect on energy saving in data centers and computer networks [37]. However, there is need for caution while lowering the energy consumption not to affect the overall data centre performance.

\section{POWER MONITORING TOOLS IN COMPUTER NETWORKS}

Many of the processor design communities have recognized that power dissipation is a first-class architectural design constraint not only for portable computers and mobile communication devices, but also for high performance superscalar microprocessors [38]. A fair amount of research efforts have been directed towards reduction of power dissipation in this high-end systems. Proposed solutions include both micro architectural and circuit-level techniques.

\subsection{Accupower}

Accupower power monitoring tool uses detailed cycle level simulation of all major data path components and interconnections that mimic the actual hardware implementation, including separate and realistic implementations of the issue queue, register files, load store queue and forwarding mechanisms.

The AccuPower tool supports built-in models for three major variants of superscalar data paths in wide use.

The AccuPower also includes well-instrumented facilities for collecting data path statistics of relevance to both power and performance at the level of bits, bytes (for data and instruction flows) within logic blocks and subsystem-level components and the entire processor.

Accupower helps in implementing of cutting edge techniques for power/energy reduction at the micro architectural level, at the logic level and circuit level, as well as techniques based on clock gating, voltage and frequency scaling to facilitate the exploration of the design space are included in AccuPower. AccuPower uses energy/power dissipation coefficients associated with the energy dissipating events within each key data path component and the interconnections. These are combined with the transition counts obtained from the micro architectural simulation component to get the overall energy/power dissipation figures. The coefficients could be estimated analytically from process specific electrical parameters by adapting tools such as CACTI [15]. A more accurate approach and one also supported in Accupower is to derive these coefficients from SPICE measurements of actual layouts of these components. The Accupower toolkit includes representative VLSI layouts of some key data path components and the dissipation coefficients estimated using SPICE for these components. Coefficients for leakage dissipations are also provided.

\subsection{Energy Audit}

Energy Audit, is a tool for measuring and inferring the power consumption of networks and individual networking devices. Energy Audit has three main components and these include the following: (i) an API for interfacing with previously deployed infrastructure management tools and thereby simplifying adoption, (ii) a community database of benchmark measurements that map device configurations to power consumption, thereby enabling accurate power consumption estimates from device queries and (iii) an auditing component that synthesizes measurements and enables network-wide energy consumption evaluation. Energy Audit is designed to flexibly combine novel power auditing capabilities with existing infrastructure monitoring tools currently used by network operators. Examples of widely used tools include Rancid [39], Net-SNMP [40], or custom management scripts, which monitor the uptime or performance of an infrastructure or provide details on configurations and status of devices. By integrating with these tools, we seek to gain access to device 
details that are sufficient to conduct a power audit without additional monitoring equipment.

With all the available tool for energy efficient we can reduce on power utilization in computer networks.

\section{INSIGHTS AND FUTURE RESEARCH}

While a lot of efforts have been devoted to the design of computer networks, a number of open questions need to be addressed regarding scalability, efficiency and effectiveness. Literature on how energy can be conserved has been reviewed. We have identified that:-

- Virtualization can be applied in computer networks with the ability to: conserve on resource consumption, and aide as a significant trade-offs between performance, Quality of service (QoS) and energy efficiency and (ii) offer insight into the manner in which energy savings can be achieved in large-scale computer networks.

- Specific plug-ins and energy-control centers for networked large-scale hardware and software can be implemented and that they can have significant impact.

The analysis and gaps presented in the sections above spur new research directions in the area of energy efficiency in computer networks for example; studies on energy usage in computer networks characterized by heterogeneity of component devices like different computer brands. The study would impact with approaches for efficient energy conservation by providing recommendations to management on what to procure for their networks.

\section{CONCLUSION}

In this state of the art survey, improvements in energy efficiency over the last two decade have been presented, giving a comprehensive account of the state of the art in the field. Specifically, literature indicates energy efficiency has continuously improved with a lot of technology advancements, energy conservation approaches, techniques and computing architectures have been diversified today than ever in the previous century, hence the difference in spending on energy costs.

We have also grouped a variety of large scale computer network architectures into three broad categories: switchcentric (the Fat-Tree, VL2 and Portland), server-centric (BCube, DCell and FiConn) and Dual centric as well as their underlying technologies into electronic, optical and hybrid electro-optical computer networks, identified their challenges and opportunities contributed from implementation. The researchers now conclude based on the discussion in the previous sections that dual centric computer networks architectures have various nice properties for practical computer networks and provide flexible choices in designing computer network architectures. This is because with as switches are becoming more programmable via software defined networking, and servers are tending to utilize specialized hardware for packet forwarding, both switches and servers will carry both packet forwarding capability and routing intelligence which is not with the other architectures.

\section{ACKNOWLEDGMENT}

Great thanks to Professor Tom Parks, Dr. Nabaasa Everest, Dr. John Businge, Mr. Kaggwa Fred, Mr. Mugonza Robert, Mr. Kawuma Simon, Mr. Nyanzi Abubark and all members of
Mbarara University distributed computing research group for their expertise and guidance during this survey.

\section{REFERENCES}

[1] M. B. Srivastava, A. P. Chandrakasan, and R. W. Brodersen, "Predictive system shutdown and other architectural techniques for energy efficient programmable computation," Very Large Scale Integration (VLSI) Systems, IEEE Transactions on, vol. 4, pp. 42-55, 1996.

[2] M. Gay, Recent advances and issues in computers: Greenwood Publishing Group, 2000.

[3] A. Berl, E. Gelenbe, M. Di Girolamo, G. Giuliani, H. De Meer, M. Q. Dang, et al., "Energy-efficient cloud computing," The computer journal, vol. 53, pp. 10451051,2010

[4] M. Bray, "Review of computer energy consumption and potential savings," Dragon Systems Software Limited (DssW), 2006.

[5] A. Hooper, "Green computing," Communication of the ACM, vol. 51, pp. 11-13, 2008.

[6] H. Wong, "EPA datacenter study IT equipment feedback summary," in Intel Digital Enterprise Group, Cited in: Report to Congress on Server and Data Center Efficiency Public Law, 2007, pp. 109-431.

[7] R. Brown, "Report to congress on server and data center energy efficiency: Public law 109-431," Lawrence Berkeley National Laboratory, 2008.

[8] E. Stanford, "Environmental trends and opportunities for computer system power delivery," in 2008 20th International Symposium on Power Semiconductor Devices and IC's, 2008.

[9] D. Wang, "Meeting green computing challenges," in Electronics Packaging Technology Conference, 2008. EPTC 2008. 10th, 2008, pp. 121-126.

[10] D. Goodin, "IT confronts the datacenter power crisis," Infoworld, October, 2006

[11] G. Lawton, "Powering down the computing infrastructure," Computer, pp. 16-19, 2007.

[12] R. R. Schmidt and H. Shaukatullah, "Computer and telecommunications equipment room cooling: a review of literature," in Thermal and Thermomechanical Phenomena in Electronic Systems, 2002. ITHERM 2002. The Eighth Intersociety Conference on, 2002, pp. 751766.

[13] J. Foley, "Google in Oregon: Mother Nature meets the data center," InformationWeek's Google Weblog, 2007.

[14] W. Forrest, J. M. Kaplan, and N. Kindler, "Data centers: how to cut carbon emissions and costs," McKinsey on business technology, vol. 14, pp. 4-13, 2008.

[15] D. T. Tohmatsu, "The Next Wave of Green IT," CFO Research Services, Boston: CFO Publishing Corp, vol. 32, 2009.

[16] R. R. Harmon and N. Auseklis, "Sustainable IT services: Assessing the impact of green computing practices," in Management of Engineering \& Technology, 2009. PICMET 2009. Portland International Conference on, 2009, pp. 1707-1717. 
[17] H. Roeth and L. Wokeck, "ICTs and climate change mitigation in emerging economies," ed: Technical report. Available at http://www.niccd.org/sites/default/files/RoethWokeckCli mateChangeMitigationICTs.pdf, accessed $2^{\text {nd }}$ June, 2016.

[18] F. Douglis, P. Krishnan, and B. Bershad, "Adaptive disk spin-down policies for mobile computers," Computing Systems, vol. 8, pp. 381-413, 1995.

[19] A. Kamilaris, B. Kalluri, S. Kondepudi, and T. K. Wai, "A literature survey on measuring energy usage for miscellaneous electric loads in offices and commercial buildings," Renewable and Sustainable Energy Reviews, vol. 34, pp. 536-550, 2014.

[20] J. Theron, "System and method for monitoring and managing power use of networked information devices," ed: Google Patents, 2002.

[21] J. Nilsson and L. Bertling, "Maintenance management of wind power systems using condition monitoring systems-life cycle cost analysis for two case studies," Energy Conversion, IEEE Transactions on, vol. 22, pp. 223-229, 2007.

[22] A. Hammadi and L. Mhamdi, "A survey on architectures and energy efficiency in data center networks," Computer Communications, vol. 40, pp. 1-21, 2014.

[23] L. Gyarmati and T. A. Trinh, "How can architecture help to reduce energy consumption in data center networking?," in Proceedings of the 1st International Conference on Energy-Efficient Computing and Networking, 2010, pp. 183-186.

[24] Y. Zhang and N. Ansari, "On architecture design, congestion notification, TCP incast and power consumption in data centers," Communications Surveys \& Tutorials, IEEE, vol. 15, pp. 39-64, 2013.

[25] M. Al-Fares, A. Loukissas, and A. Vahdat, "A scalable, commodity data center network architecture," ACM SIGCOMM Computer Communication Review, vol. 38, pp. 63-74, 2008.

[26] C. Guo, H. Wu, K. Tan, L. Shi, Y. Zhang, and S. Lu, "Dcell: a scalable and fault-tolerant network structure for data centers," ACM SIGCOMM Computer Communication Review, vol. 38, pp. 75-86, 2008.

[27] A. Greenberg, P. Lahiri, D. A. Maltz, P. Patel, and S. Sengupta, "Towards a next generation data center architecture: scalability and commoditization," in Proceedings of the ACM workshop on Programmable routers for extensible services of tomorrow, 2008, pp. $57-62$

[28] R. Niranjan Mysore, A. Pamboris, N. Farrington, N. Huang, P. Miri, S. Radhakrishnan, et al., "Portland: a scalable fault-tolerant layer 2 data center network fabric," in ACM SIGCOMM Computer Communication Review, 2009, pp. 39-50.

[29] M. Al-Fares, S. Radhakrishnan, B. Raghavan, N. Huang, and A. Vahdat, "Hedera: Dynamic Flow Scheduling for Data Center Networks," in NSDI, 2010, pp. 19-19.

[30] A. Greenberg, J. R. Hamilton, N. Jain, S. Kandula, C. Kim, P. Lahiri, et al., "VL2: a scalable and flexible data center network," in ACM SIGCOMM computer communication review, 2009, pp. 51-62.
[31] C. Guo, G. Lu, D. Li, H. Wu, X. Zhang, Y. Shi, et al., "BCube: a high performance, server-centric network architecture for modular data centers," ACM SIGCOMM Computer Communication Review, vol. 39, pp. 63-74, 2009.

[32] D. Chernicoff, The shortcut guide to data center energy efficiency: Realtimepublishers. com, 2009.

[33] A. Greenberg, J. Hamilton, D. A. Maltz, and P. Patel, "The cost of a cloud: research problems in data center networks," ACM SIGCOMM computer communication review, vol. 39, pp. 68-73, 2008.

[34] B. Heller, S. Seetharaman, P. Mahadevan, Y. Yiakoumis, P. Sharma, S. Banerjee, et al., "ElasticTree: Saving Energy in Data Center Networks," in NSDI, 2010, pp. 249-264.

[35] Y. Shang, D. Li, and M. Xu, "Green Routing in Data Center Network: Modeling and Algorithm Design," in Proceedings of the first ACM SIGCOMM workshop on Green networking.

[36] D. Kliazovich, P. Bouvry, and S. U. Khan, "GreenCloud: a packet-level simulator of energy-aware cloud computing data centers," The Journal of Supercomputing, vol. 62, pp. 1263-1283, 2012.

[37] 화정서 and 호원김, "On Dynamic Voltage Scale based Protocol for Low Power Underwater Secure Communication on Sensor Network," Journal of the Korea Institute of Information and Communication Engineering, vol. 18, pp. 586-594, 2014.

[38] S. Nedevschi, L. Popa, G. Iannaccone, S. Ratnasamy, and D. Wetherall, "Reducing Network Energy Consumption via Sleeping and Rate-Adaptation," in NSDI, 2008, pp. 323-336.

[39] T. Mudge, "Power: A first-class architectural design constraint," Computer, pp. 52-58, 2001.

[40] D. Brooks, V. Tiwari, and M. Martonosi, Wattch: a framework for architectural-level power analysis and optimizations vol. 28: ACM, 2000.

[41] S. J. Wilton and N. P. Jouppi, "An enhanced access and cycle time model for on-chip caches," 1993.

[42] D. Ponomarev, G. Kucuk, and K. Ghose, "AccuPower: An accurate power estimation tool for superscalar microprocessors," in Proceedings of the conference on Design, automation and test in Europe, 2002, p. 124.

[43] A. Dhodapkar, C. H. Lim, G. Cai, and W. R. Daasch, "Tem2p2est: A thermal enabled multi-model power/performance estimator," in Power-Aware Computer Systems, ed: Springer, 2000, pp. 112-125

[44] S. Herbert and D. Marculescu, "Analysis of dynamic voltage/frequency scaling in chip-multiprocessors," in Low Power Electronics and Design (ISLPED), 2007 ACM/IEEE International Symposium on, 2007, pp. 3843.

[45] D. Li, J. Wu, Z. Liu, and F. Zhang, "Dual-Centric Data Center Network Architectures," in Parallel Processing (ICPP), 2015 44th International Conference on, 2015, pp. 679-688. 